\title{
Burst Assembly Framework for High Speed Optical Network
}

\author{
H. Saini ${ }^{*}$, A.K. Garg ${ }^{2}$ \\ ${ }^{1}$ Assistant Professor, D.C.R.U.S.T. Murthal, Sonepat, India. \\ ${ }^{2}$ Professor, D.C.R.U.S.T. Murthal, Sonepat, India. \\ *Corresponding author E-mail:himanshi.4887@gmail.com
}

\begin{abstract}
Optical Burst Switching (OBS) is promising technique to support requirements of high speed optical networks. OBS network ingress node assembles packets into bursts. Burst assembly criteria have to be optimally chosen based on network requirements. In this paper, burst assembly framework is presented which can support optimal time/size value selection during burst formation. It is observed that, network with high timeout supports high Transmission Control Protocol (TCP) throughput for a range of burst size. For lower burst timeout values, throughput performance degrades only for small size bursts and further reduction in timeout threshold degrades throughput for range of burst size.
\end{abstract}

Keywords: Burst assembly, throughput, timeout, burst size.

\section{Introduction}

Dense Wavelength Division Multiplexing (DWDM) technology has resulted into enormous data rates per wavelength in high speed optical networks. Switching technology used in an optical network has direct impact on bandwidth utilization in a network. OBS has emerged as promising technique for high speed networks carrying busty traffic. It can handle high speed busty internet traffic efficiently than Optical Circuit Switching (OCS) and Optical Packet Switching (OPS). OBS has combined advantages of OCS like buffer avoidance and OPS like low latency, high bandwidth utilization and traffic adaptively. Separation of control and data packets in time and wavelength allows OBS to eliminate buffering issues [1]. Time separation between control packet and data burst is called Offset. Burst assembly, channel scheduling, offset time, Routing and Wavelength Assignment (RWA), contention resolution and signalling mechanism are major factors which decide functioning of OBS network [2]. OBS network is comprised of core and ingress/egress edge nodes as shown in figure 1. Ingress edge nodes are responsible for grouping set of data units like IP packets into a burst and grouping is performed based on assembly technique i.e. time based, size based or hybrid adopted in the network [3].

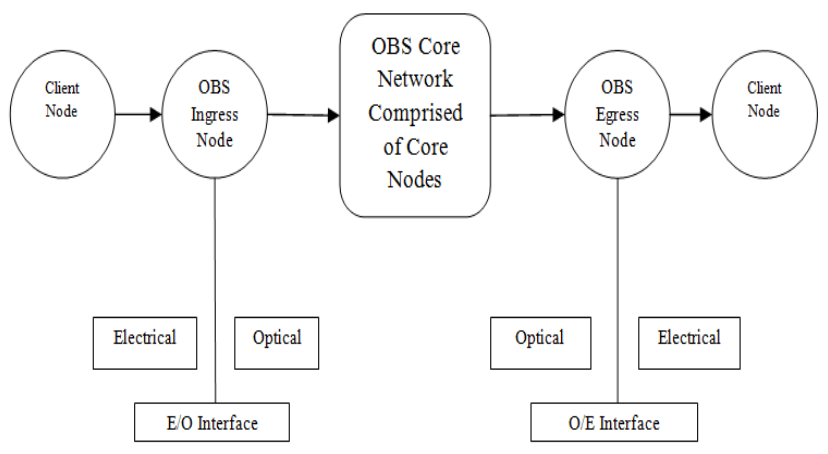

Fig. 1: OBS network basic blocks
Edge nodes in OBS network are responsible for data assembly, Burst Header Control Packet (BHCP) generation, signalling and routing. Data burst is separated from BHCP by OFFSET time units. Information such as burst size, offset time, source and destination address of burst is carried by BHCP. Higher value of offset time reduces the issue of burst contention [4]. Assembly techniques at ingress node can be based on size of burst, delay of first packet in burst. Hybrid burstifier generates a burst when either of size threshold or timeout limit has reached [5]. For large burst size threshold limit, hybrid assembly technique performs as timer based assembly technique. Reservation in OBS networks is performed through Just In Time (JIT), Just Enough Time (JET) or Horizon based techniques. JIT or Immediate Reservation reserves resources for the incoming burst as soon as the BHCP arrives, if resources are not free at that time then set up message is rejected and burst corresponding to that setup message is lost. JIT protocol implementation is easy as compared to JET and Horizon as it does not incorporate void filling or scheduling mechanisms [6]. JET or Delayed Reservation protocol reserves resources for data burst just before arrival of first bit of burst. At BHCP arrival, if it is estimated that required resources cannot be provided at appropriate time estimated for arrival of data burst, then setup message is rejected [7].

This paper addresses the issue of burst assembly and its impact on TCP throughput. Some of the previous investigations and proposals on issues related to OBS networks are presented in Section 2. Section 3 presents the simulation environment. Simulation tool, parameters, test network configuration are covered in this section. Results are discussed in section 4. Average and instantaneous throughput curves are plotted in this section for range of burst size and timeout values. Section 5 concludes the investigations performed for burst assembly framework

\section{Related Work}

Major concerns for OBS networks are burst assembly technique selection, thresholds to be adopted for time or size based assembly 
technique, reservation technique selection, routing and wavelength assignment, resource scheduling, contention resolution. All the concerns get aggravated as traffic becomes still bustier. Several OBS network concerns and solutions have been investigated and proposed in literature. Pavani et. al. [8] investigated OBS network performance under TCP-Reno and TCP-Vegas. Burst delivery ratio has been estimated for different values of hop delay, sizes of burst and maximum flow queue. Debnath et. al. [9] have presented simulation results for performance of burst switching under self similar IP traffic. Time limit based assembly is observed to have highest probability of burst loss when compared with burst size limit and mixed policy. Sodhatar et. al. [10] compared TCP variants in OBS network and concluded that network performance in terms of throughput is better for TCPReno as compared to TCP-Tahoe. Mohd Sam et. al. [11] proposed a FDL approach and core node architecture based on wavelength assignment and pre-emption. Modelled and plotted E2E Delay, Bandwidth Utilization and Throughput for determining QoS of the proposed approach. Proposed approach offer better performance as compared to conventional approaches. Soni et. al. [12] discussed the impact of offset time setting on performance of OBS network. Large offset time is required to maintain the performance of OBS network for heavy load. They have stressed upon the need of dynamic offset time setting instead of fixed offset. Coulibaly et. al. [13] stressed upon importance of security of burst control packet and developed a solution to tackle attacks which redirect the data bursts. They have investigated various methods for control packet protection. Reduction in burst loss and increase in throughput has been observed with proposed security mechanism. Coulibaly et. al. [14] have shown considerable reduction in burst loss with slotted OBS. Imran et. al. [15] modelled switch architecture and tested it under different network load to examine TCP over OBS performance. Aguas et. al. [16] have shown the impact of link, node, channel and fibre failure on performance of OBS network. The analysis is performed under high and medium network load conditions under a range of failure probability values. It has been shown that node failures cause drastic loss to OBS networks under heavy load.

\section{Simulation Details}

The simulation platform nOBS [17] have been used in the present investigation. nOBS is developed by extending Network Simulator 2 (NS2) [18] existing architecture by Guray Gurel, Onur Alparslan and Ezhan Karasan [17]. Test network structure with 14 nodes and 20 links as shown in figure 2 is used in this study. 6 nodes (node number $0,1,2,3,4,5$ ) are configured as optical nodes. These nodes have common functionality as core or edge nodes. Node 0 and 5 are ingress edge nodes. Node 4 is egress edge node. JET reservation protocol has been incorporated in nOBS. Maximum size of packets/ Maximum Transmission Unit entering the burstifier (max_MTU) is 1040 bytes. Nodes 1, 2 and 3 (shown in orange colour in figures) comprise core OBS network and nodes 0,4 and 5 (shown in red colour in figures) are edge OBS nodes. Simultaneous File Transfer Protocol (FTP) flow as shown in figure 3 from node 6 and 7 is simulated at $10^{\text {th }}$ second of simulation interval. For simulation interval 0-10 seconds, FTP flow from node 6 continues and for interval 10-20 seconds, simultaneous FTP flow from node 6 and 7 is simulated

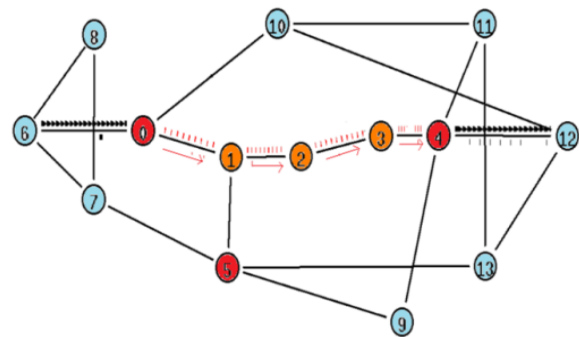

Fig. 2: Test network (optical nodes: 0, 1, 2, 3, 4, 5, transmitting access node: 6 )

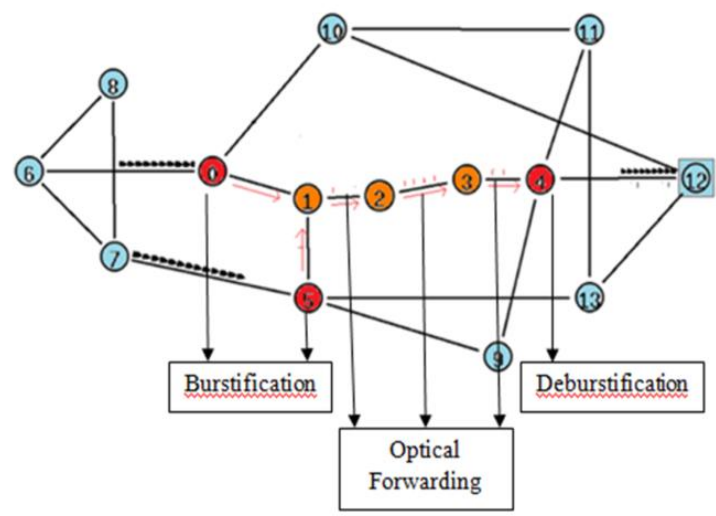

Fig. 3: Test network (optical nodes: 0, 1, 2, 3, 4,5, transmitting access nodes: 6 and 7)

Figure 4 is illustrating the network segments involved in routing. Source routing is selected with routes as 6-0-1-2-3-4-12 and 7-51-2-3-4-12 for both the source nodes 6 and 7 respectively. $0-1,5-$ $1,1-2,2-3$ and 3-4 links are configured as optical links

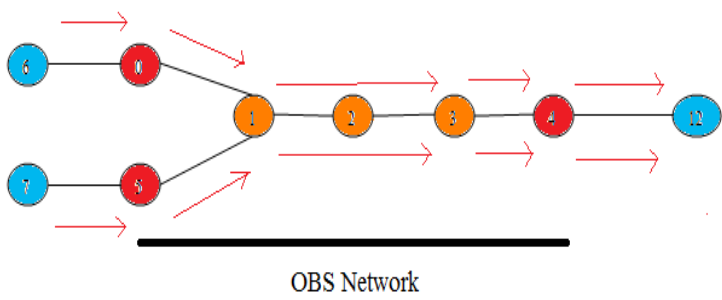

Fig. 4: Route from source nodes $(6,7)$ to destination node (12)

\section{Results and Discussions}

TCP throughput is measured at node 12 for 20 second simulation time. Simultaneous transmission form node 6 and 7 starts at $10^{\text {th }}$ second simulation instant and continues till end of simulation, prior to this time instant only node 6 is transmitting. Figure 5 shows the average throughput plot with respect to burst size for various values of timeout. It is observed that throughput remains nearly constant for burst size greater than 100 packets for each timeout value and maximum value is obtained at $7 \mathrm{~ms}$ timeout. Average value of throughput reduces for small and large timeout values. With increase in burst size throughput attains a constant value after a threshold value of burst size.

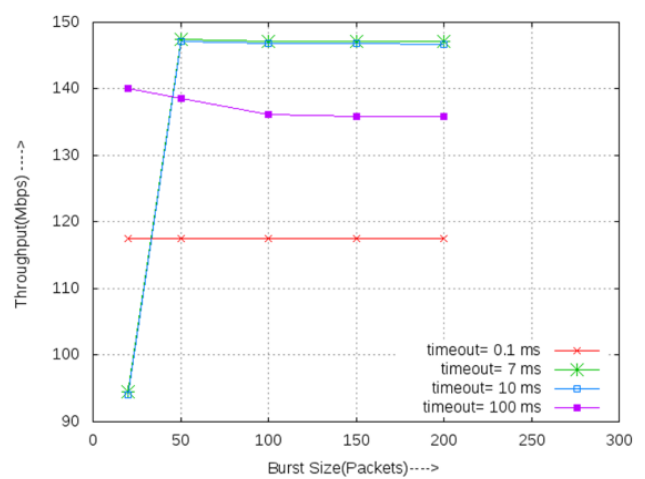

Fig. 5: Average throughput vs. burst size for range of timeout values

Instantaneous throughput curves with respect to simulation time are shown in figure 6-9. Figure 6 illustrates throughput variation for $0.1 \mathrm{~ms}$ timeout. Reduction in throughput is observed for all the considered burst size after simultaneous transmission from nodes 6 and 7 starts at 10 second simulation time instant. For higher timeout value of $7 \mathrm{~ms}$ as shown in figure 7 , throughput increases continuously except for burst size of 20 packets whose throughput falls after both nodes 6 and 7 starts transmission at $10 \mathrm{sec}$. Same throughput variation is observed for timeout of $10 \mathrm{~ms}$ as observed 
from figure 8 . Figure 9 shows throughput variation for still higher value of timeout 100ms.[19] In this case, throughput is highest for least burst size considered and none of the burst size cases show reduction in throughput after simultaneous transmission of data at 10 second simulation time instant. [20]

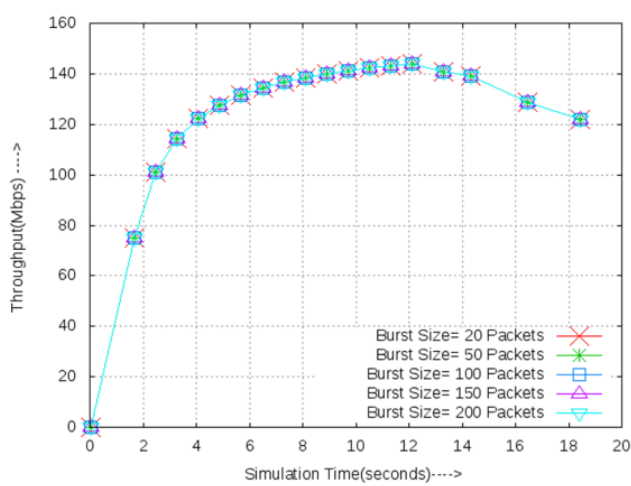

Fig. 6: Instantaneous throughput for a range of Burst Size at Timeout= 0.1 msec

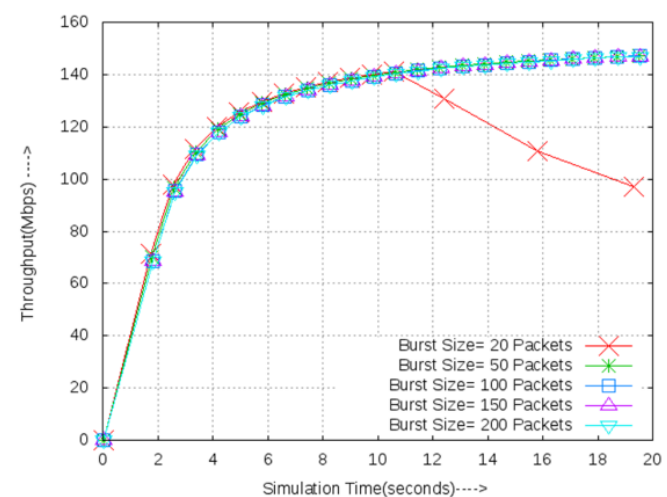

Fig. 7: Instantaneous throughput for a range of burst size at timeout= 7 msec

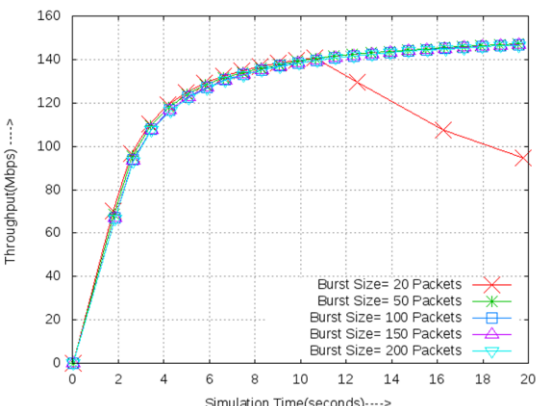

Fig. 8: Instantaneous throughput for a range of burst size at timeout $=10$ msec

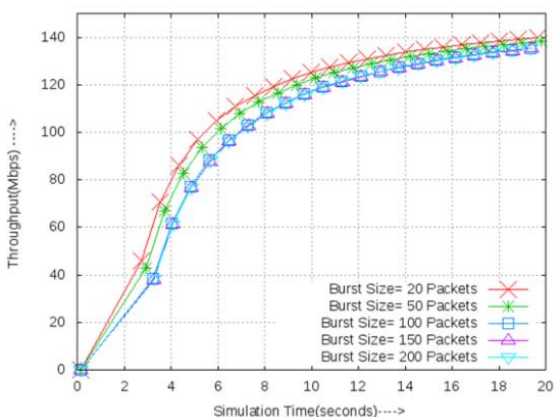

Fig. 9: Instantaneous throughput for a range of burst size at timeout $=100$ msec

\section{Conclusion}

OBS combines merits of OPS and OCS. High bandwidth utilization, low set up latency, high traffic adaptively and separation of optical and electronic control have made OBS, the promising technology for DWDM future high speed networks. To extract advantages out of OBS technology, various OBS network concerns have to be addressed. In this paper, an overview of basic OBS concepts is presented and burst assembly techniques have been discussed. TCP throughput is examined for various combinations of burst timeout and size. For higher timeout value, lowest considered burst size attains highest throughput. Low timeout values leads to same reduction in throughput irrespective of burst size. Selection of optimum burst timeout and size value is imperative in order to meet Quality of Service (QoS) of WDM optical networks.

\section{References}

[1] Andrews JG, Buzzi S, Choi W, Hanly SV, Lozano A, Soong AC \& Zhang JC, "What will 5G be?", IEEE Journal on selected areas in communications, Vol.32, No.6, (2014), pp.1065-1082.

[2] Xin Y, Battestilli L \& Karmous-Edwards G, "Generic optica network provisioning services to support emerging grid applications", Fourth International Conference on Broadband Communications, Networks and Systems, (2007), pp.131-140.

[3] Yahaya C, Latiff A \& Mohamed AB, "A review of routing strategies for optical burst switched networks", International Journal of Communication Systems, Vol.26, No.3, (2013), pp.315-336.

[4] Choudhury S, Nair V \& Mal AK, "Routing scheme for OBS networks", Journal of Optical Communications and Networking, Vol.4, No.10, (2012), pp.799-811.

[5] Akar N \& Karasan E, "Exact calculation of blocking probabilities for bufferless optical burst switched links with partial wavelength conversion", IEEE First International Conference on Broadband Networks, (2004), pp.110-117.

[6] Triay Marquès J, Rubio J \& Cervelló Pastor C, "An Optical Burs Switching Control Plane Architecture and its Implementation", 12th Open European Summer School EUNICE, Vol.1, (2006).

[7] Teng J \& Rouskas GN, "A detailed analysis and performance comparison of wavelength reservation schemes for optical burst switched networks", Photonic Network Communications, Vol.9, No.3, (2005), pp.311-335

[8] Pavani KR \& Sreenath N, "Performance Evaluation of TCP NewVegas and TCP Newreno on Burstification in an OBS Network", Computer Networks \& Communications (NetCom), (2013), pp.185-194.

[9] Figgatt C, Maslov D, Landsman KA, Linke NM, Debnath S \& Monroe C, "Complete 3-qubit Grover search on a programmable quantum computer", Nature communications, Vol.8, No.1, (2017).

[10] Sodhatar SH, Patel RB \& Dave, J. V. (2012, May). Throughput Based Comparison of Different Variants of TCP in Optical Burst Switching (OBS) Network. IEEE International Conference on Communication Systems and Network Technologies (CSNT), (2012), pp.371-376

[11] Sam SM, Daud SM, Kamardin K \& Maarop N, "Study of Qos Performance in Optical Burst Switched Networks (OBS)", Indian Journal of Science and Technology, Vol.9, No.48, (2016).

[12] Soni A \& Nath Singh Y, ICEIT Conference on Advances in Mobile Communications, Networking and Computing, New Delhi, (2017)

[13] Coulibaly Y. IEEE International Broadband and Photonics Conference (IBP), Bali, (2015)

[14] Coulibaly Y. IEEE 12th Malaysia International Conference on Communications (MICC), Kuching, Malaysia, (2015).

[15] Imran M, Collier M, Landais P \& Katrinis K, "Performance Evaluation of TCP over Optical Burst-Switched Data Center Network", IEEE 18th International Conference on Computational Science and Engineering (CSE), (2015), pp.51-57.

[16] Aguas E, Calderón J \& Puerto G, "Workshop on Engineering Applications", International Congress on Engineering (WEA), Bogota, (2015).

[17] Gurel G, Alparslan O \& Karasan E, "nOBS: an ns2 based simulation tool for performance evaluation of TCP traffic in OBS networks", Annales des telecommunications, Vol.62, No.5-6, (2007), pp.618-637.

[18] The Network Simulator: NS2, https://www.isi.edu/nsnam/ns/

[19] Z Yesembayeva (2018). Determination of the pedagogical conditions for forming the readiness of future primary school teachers, Opción, Año 33. 475-499

[20] G Mussabekova, S Chakanova, A Boranbayeva, A Utebayeva, K Kazybaeva, K Alshynbaev (2018). Structural conceptual model of forming readiness for innovative activity of future teachers in general education school. Opción, Año 33. 217-240 